\title{
Radiation therapy dose prediction for left-sided breast cancers using two-dimensional and three-dimensional deep learning models
}

\author{
Natasha Hedden ${ }^{a, b, *}$, Heping $\mathrm{Xu}^{\mathrm{b}}$

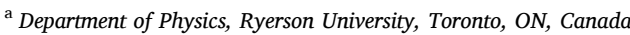 \\ ${ }^{\mathrm{b}}$ Department of Medical Physics, Walker Family Cancer Centre, St. Catharines, ON, Canada
}

\section{A R T I C L E I N F O}

\section{Keywords:}

Machine learning

Deep learning

Dose prediction

Breast cancer

\begin{abstract}
A B S T R A C T
Purpose: To develop a deep learning model capable of producing clinically acceptable dose distributions for leftsided breast cancers for 3D-CRT while exploring the use of two-dimensional versus three-dimensional anatomical data.

Methods: Two deep learning models, a two-dimensional and three-dimensional model, based on U-net architecture were trained to predict dose distribution given anatomical information and dose prescription. The input consists of 6 channels including the patient CT along with binary masks for four OARs and one covering the volume receiving 95\% dose (based on the clinical plan). A training set of 120 patients was compiled and used with 5 -fold cross validation. The best performing model from the 5 folds was analyzed with a test set of 25 patients using cumulative DVH, mean differences in mean dose to OARs represented by box plots, and V20 of the left lung.

Results: We have shown that both models are capable of producing clinically acceptable dose distributions, with the 3D outperforming the $2 \mathrm{D}$ model. The average dose difference for mean dose is within $0.02 \%$ of the dose prescription for both models. The V20 from the predicted dose distributions are comparable with the V20 from clinical plans, where predictions tend to be slightly under.

Conclusions: Based on the results, the models could be implemented clinically to produce dose distributions that can be used as a reference to ensure the most ideal plan is used. Each prediction is patient-specific while requiring minimal time and information creating a new standard in plan quality without hindering the planning process.
\end{abstract}

\section{Introduction}

Radiation therapy following breast conserving surgery has become a routine part of treatment in women with breast cancer, reducing the risk of local recurrence [1-3]. While the technology used to perform these treatments has advanced, treatment planning has moved forward at a slower rate as plans have become more complex while relying heavily on the skills and decisions of individual planners $[4,5]$. This can result in a large delay in the development and finalization of plans as well as a lot of variation in plan quality [4-7]. This delay can have very negative effects resulting in the subsequent delay of treatment [8,9] which, in breast cancer, has been seen to compromise local control significantly increasing the risk of local recurrence [10-12]. Planning the irradiation of left-sided breast cancer in particular requires high caution as it can result in unintended cardiac irradiation [13-15]. These plans center around producing an ideal dose distribution maximizing dose to the planning treatment volume (PTV) while minimizing dose to the organsat-risk (OARs), but DVH-based planning lacks spatial information in the cumulative DVH diagrams requiring production of dose distributions increasing the amount of intervention necessary and time to produce plans $[16,4]$.

The implementation of artificial intelligence (AI) in treatment planning can reduce the time needed to produce a clinically acceptable dose distribution by removing the need for human intervention $[4,5,17,18]$. The development of convolutional neural networks (CNN) in deep learning has allowed for better analysis of visual data such as patient anatomy and thus allowed for application in this field $[4,5]$. CNNs have been used to successfully predict dose distributions from patient anatomy for prostate [5,19], head-and-neck [16,18,20,21], and lung cancer [17]. This shows promise for the use of AI in treatment

\footnotetext{
* Corresponding author at: Department of Physics, Ryerson University, Toronto, ON, Canada.

E-mail address: natasha.hedden@ryerson.ca (N. Hedden).
} 

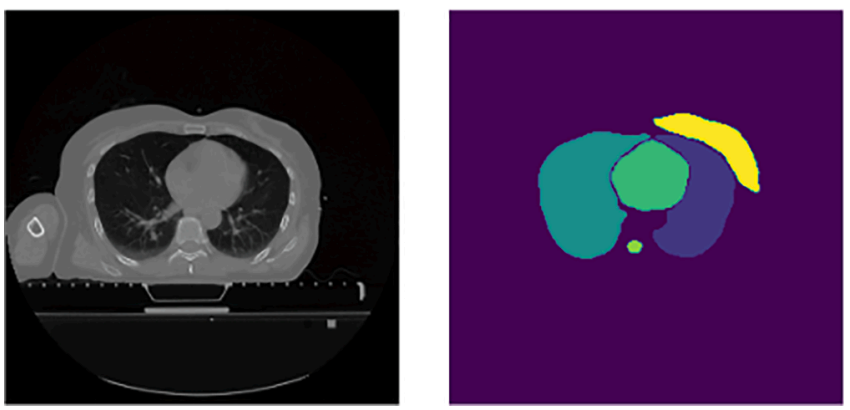

Fig. 1. The model input included patient CT images with binary masks of 5 contoured regions of interest including the left and right lungs, heart, spinal canal, and the area covered by the $95 \%$ isodose curve.

planning, but many of these papers use either only a two-dimensional or three-dimensional models.

In this work, we develop and compare a two-dimensional and threedimensional deep learning model for dose distribution prediction of leftsided breast cancers based on the U-net architecture for efficient and precise results. The model is trained on patient anatomy and dose prescription for patients with varying beam configuration and beam energies based on three-dimensional conformal radiation therapy (3DCRT) plans. It is then analyzed using cumulative dose-volume histograms, mean differences in mean dose to each region of interest weighted by the dose prescription, and V20. In producing a model capable of predicting clinically acceptable distributions, we are able to provide physicians and planners with a patient-specific reference point of an ideal dose distribution to aim for when developing plans. The dose distributions are quickly produced with minimal information ensuring that the most optimal plans are used without compromising efficiency in the planning process and creating a new standard in the quality of plans.

\section{Methods}

\subsection{Patient data and preprocessing}

A total of 145 left-sided breast cancer patients were retrospectively selected for this study, all treated using 3D-CRT (forward planning) from 2017 to 2020 with 2 or 4 tangential beams having energies of 6,10 , and/ or $18 \mathrm{MV}$. Each patient had a prescribed dose of $42.56 \mathrm{~Gy}$ in 16 fractions. Patient CT images, contoured structures, and their clinically delivered dose distributions were retrieved from the Pinnacle treatment planning system as Digital Imaging and Communications in Medicine (DICOM) files. This data was processed using a combination of the software 3D Slicer [22] with the SlicerRT toolkit [23] and the scientific computing packages NumPy $[24,25]$ and pydicom [26]. These contoured structures include left lung, contralateral lung, heart, spinal canal, and the area covered by the $95 \%$ isodose curve (Fig. 1). Majority of structures were contoured by trained treatment planners with some of the contralateral lung and spinal canal structures done through auto-segmentation and subsequently reviewed by the first author. The structure containing the 95\% dose area was used to represent the planning treatment volume (PTV) due to limitations of the clinical data available.

For the two-dimensional model, each patient volume was resampled to $256 \times 256 \times 128$ pixels through linear interpolation and separated into 128 slices. This results in a total 18,560 slices for training, validation, and testing. The contoured organs-at-risk (OAR) were separated into individual binary masks with a value of 1 indicating a pixel containing the corresponding structure and 0 indicating a pixel containing background. The $95 \%$ dose area was also set as a binary mask, but used the dose prescription rather than a value of 1 . The CT images were normalized with min-max normalization to have values ranging $0-1$ to ensure they are on a similar scale to the masks such that they provide equal contributions to the model avoiding bias. The CT images were given as input to the model with each binary mask included as its own channel for a total of 6 channels. Data augmentation was used to prevent overfitting where approximately $30 \%$ of samples were randomly flipped along the vertical axis. The output of the model is the slice-by-slice dose distribution prediction as the model learns to interpret the exact dose values as the pixel values.

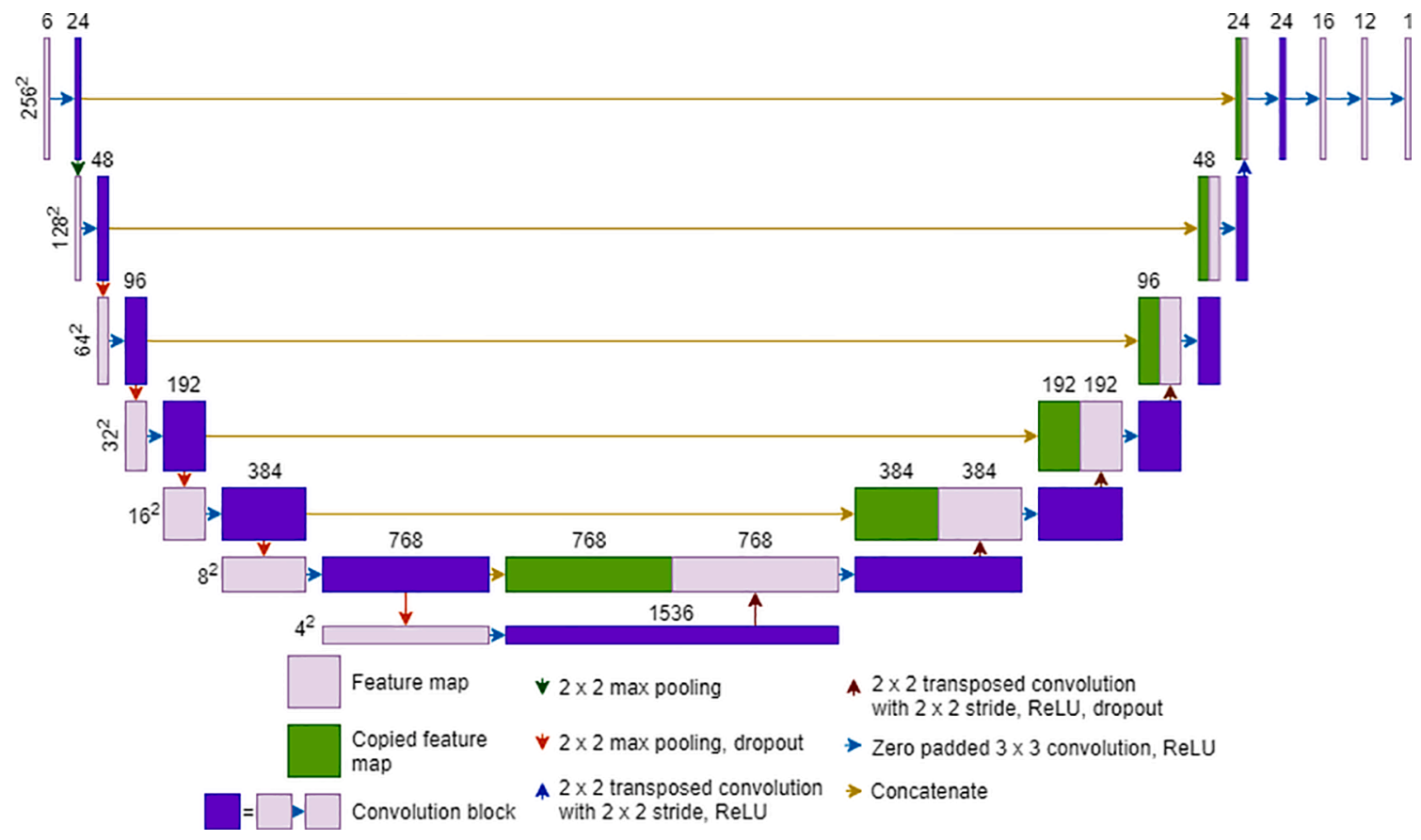

Fig. 2. The architecture of the two-dimensional U-net with 7 levels. The numbers on the left correspond to the area of the multi-channel feature maps while the numbers on the top indicate the number of channels. 


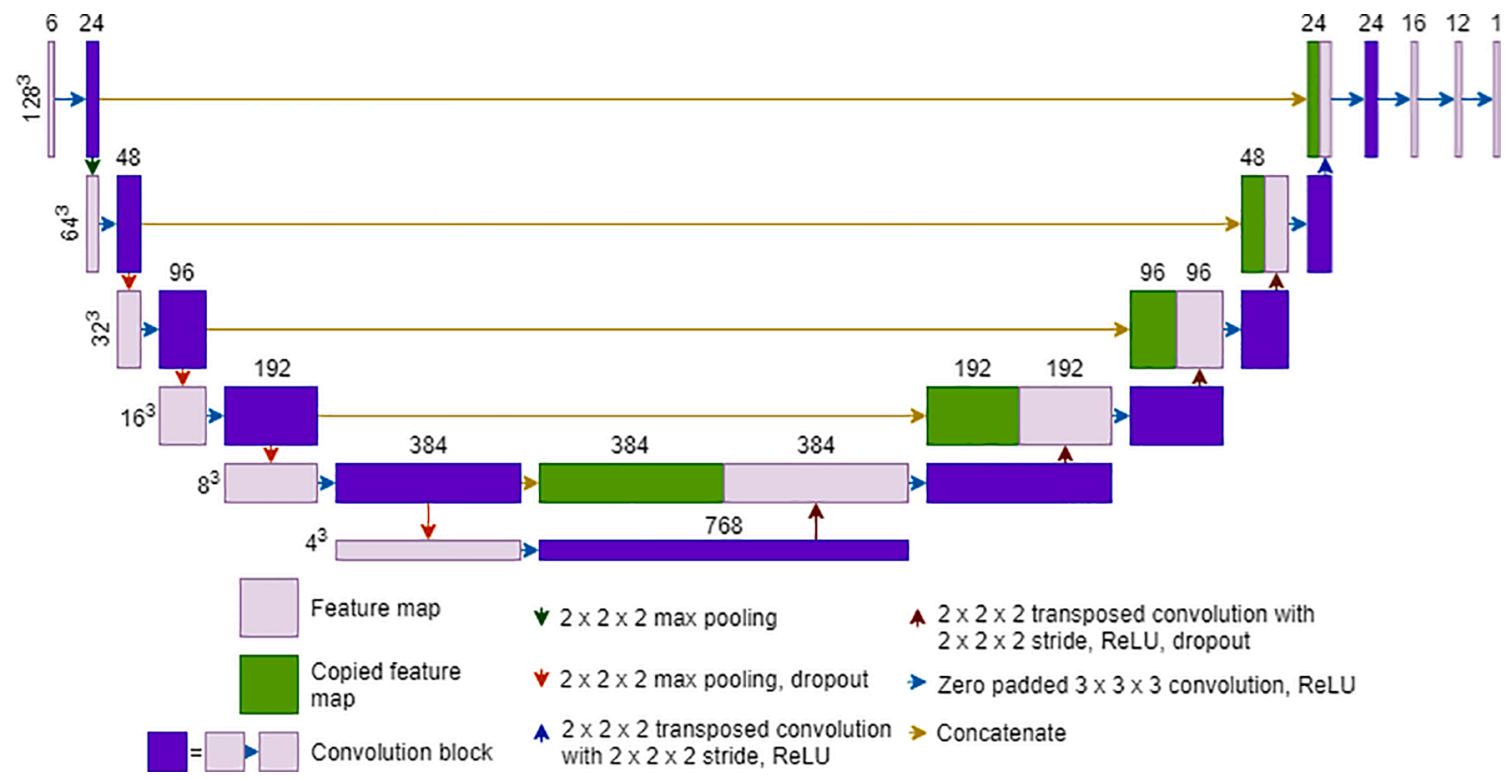

Fig. 3. The architecture of the three-dimensional U-net with 6 levels. The numbers on the left correspond to the area of the multi-channel feature maps while the numbers on the top indicate the number of channels.

The data for the three-dimensional model underwent identical processing. Randomized sub-volumes of $128 \times 128 \times 128$ pixels containing the CT images and binary masks were then given as input to the model which allows for inherent data augmentation through translation of samples. A voxel-wise dose distribution prediction is output from the model.

\subsection{Model architecture}

The two-dimensional model consists of a 7-level U-net [27] architecture with an additional three CNN layers at the end as seen in Fig. 2. This architecture allows for fast training over the images without sacrificing precision through the use of several skip connections. The additional CNN layers allow for a smoother decrease in the number of filters from 24 to 1 with improved accuracy. The seven levels were chosen maximizing computational use and reducing the input data from $256 \times 256$ to $4 \times 4$ with a convolution kernel size of $3 \times 3$ and max pooling and transposed convolution kernel size of $2 \times 2$. The model was tested with various rates of dropout and it was found that it performed best with a maximum dropout rate of 0.5 at the bottom decreasing by 0.1 for every level up. The dropout is applied after the max pooling and transposed convolutional layers. Mean squared error (MSE) was chosen

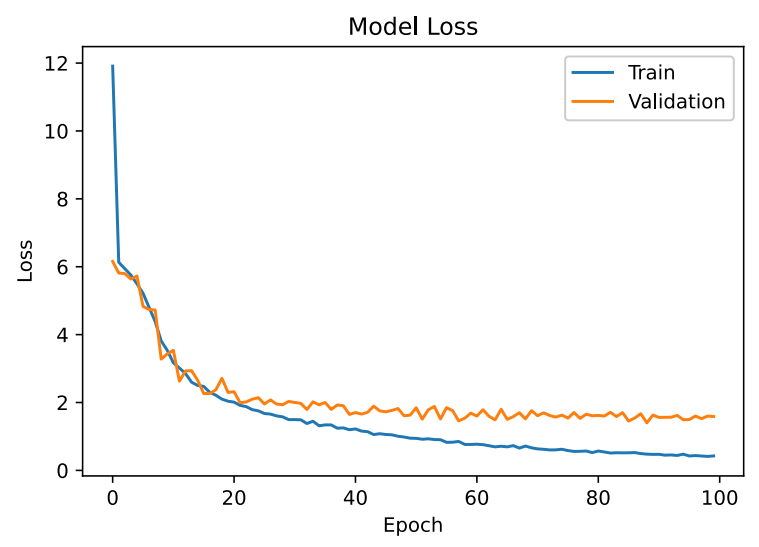

(a) as the loss function, which is the average squared difference between the predicted value, $x_{i}$, and the actual value, $\widehat{x}_{i}$, for a total $\mathrm{N}$ data points as seen in the equation: $M S E=\frac{1}{N} \sum_{i=1}^{N}\left(x_{i}-\widehat{x}_{i}\right)^{2}$. Adam [28], short for adaptive moment estimation, was used as the optimizer to update parameters with an initial learning rate of 0.0001 . Rectified linear unit (ReLU) was used as the activation function following each convolution.

The three-dimensional model contains similar structure with a 6level U-net architecture and an additional three CNN layers at the end shown by Fig. 3 . A convolution kernel size of $3 \times 3 \times 3$ was used with max pooling and transposed convolution kernel size of $2 \times 2 \times 2$. The models were built using the python library Keras [29] with Tensorflow [30] as the backend. Both models were trained on dual NVIDIA Geforce RTX 2080 Ti GPUs with 11 GB RAM each using data parallelism for the two-dimensional model and model parallelism for the three-dimensional model.

\subsection{Training and evaluation}

A set of 25 patients were put aside for testing leaving 120 patients for training and validation. To get use of the full dataset, we used 5-fold cross validation for analysis which splits the training data into 5 groups of equal size. We then train the model on the first 4 groups while

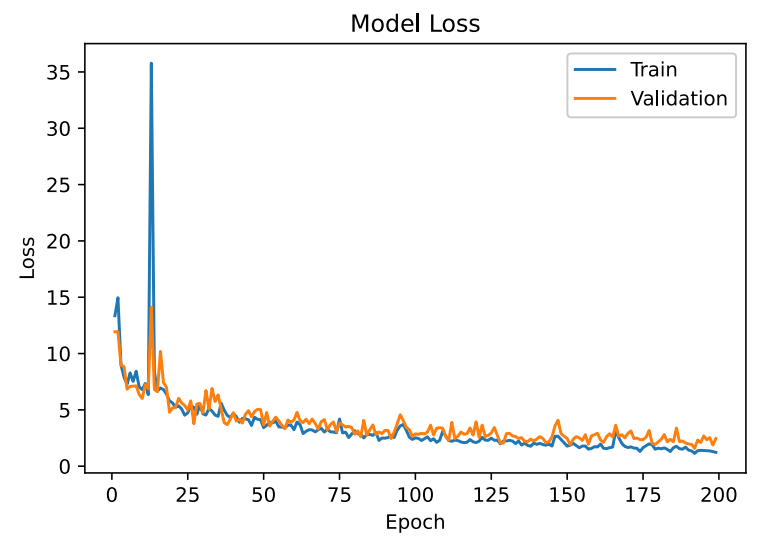

(b)

Fig. 4. The model loss as a function of epochs for the (a) 2D model and (b) 3D model. 

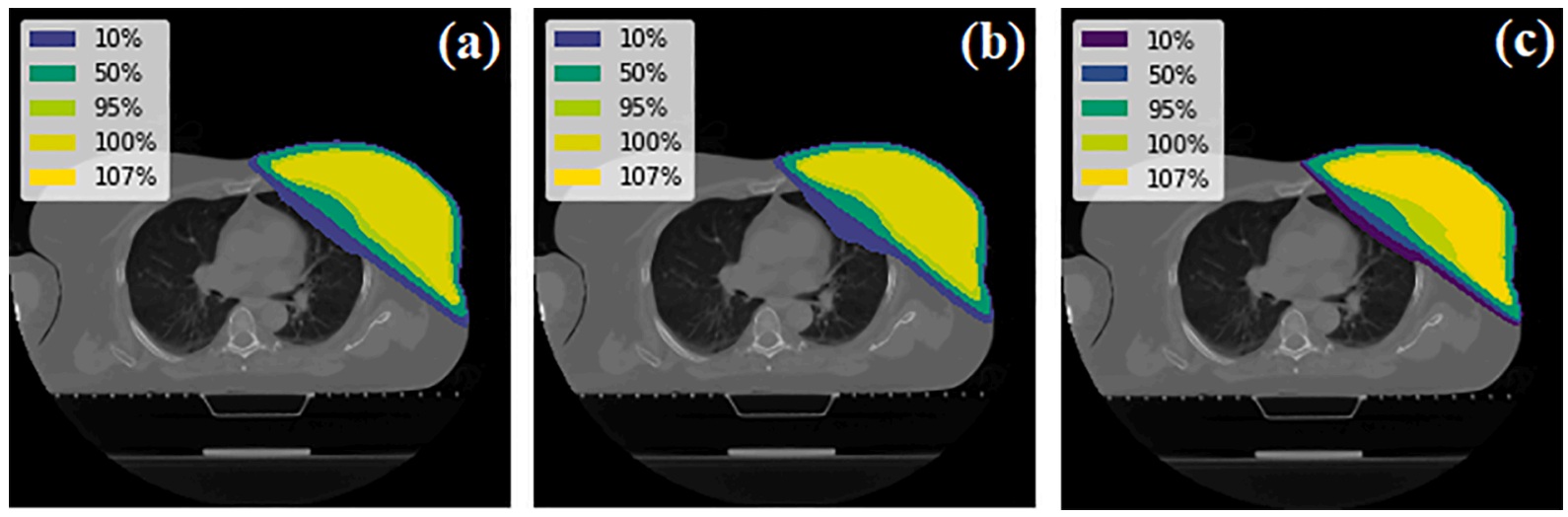

Fig. 5. The (a) 2D, (b) 3D, and (c) clinically used dose distributions weighted by the dose prediction for a random slice of the CT volume of patient A.
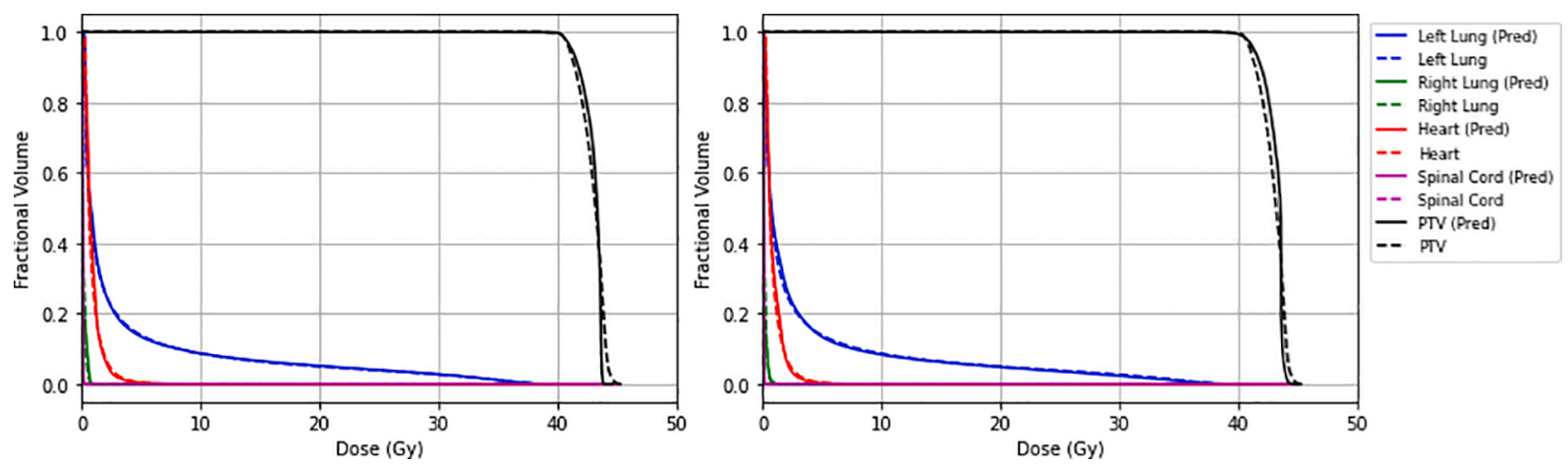

Fig. 6. The cumulative DVH graphs comparing the clinically used results with the 2D model on the left and 3D model on the right for patient A.

using the last to perform validation, which is known as fold 1 producing the first model. Fold 2 then consists of using groups 1 and $3-5$ to train while using group 2 for validation producing a second model. This process repeats until the model has been trained and validated over all data allowing for usage of the entire dataset while preventing the model from being tested on previously seen data. A batch-size of 32 slices was chosen as input for the two-dimensional model with a batch-size of 2 sub-volumes for the three-dimensional model. The overall performance of the chosen architecture is then given by an average of the training and validation loss for all 5 models. The model with the lowest mean squared error on both validation and test data, and thus the best generalized model, was then used to produce dose predictions which were used for visualization and evaluated using box plots to represent the mean differences in mean dose for each region of interest weighted by the dose prescription as seen in the equation: Dose $e_{\text {diff }}=\frac{D_{\text {true }}-D_{\text {pred }}}{D_{\text {prescription }}} \times 100$, and box plots comparing V20 of the dose predictions from both models with the V20 from clinically used plans where V20 is the relative volume of the left lung receiving $\geqslant 20 \mathrm{~Gy}$.

\section{Results}

The average mean squared error over all 5 folds was graphed as a function of epochs for both training and validation in Fig. 4 where the 2D model was run for 100 epochs and the 3D model was run for 200 epochs.

Predictions were produced for all patients in the test set where to visualize the performance of the models, the dose distributions of two randomly selected patients are compared here. Fig. 5 displays the comparison of dose distributions weighted by the prescription dose for patient A while Fig. 6 shows a comparison of the cumulative DVH for the 2D and 3D model against the clinically predicted DVH. The results for patient B can be found in the appendix (Figs. A1 and A2).

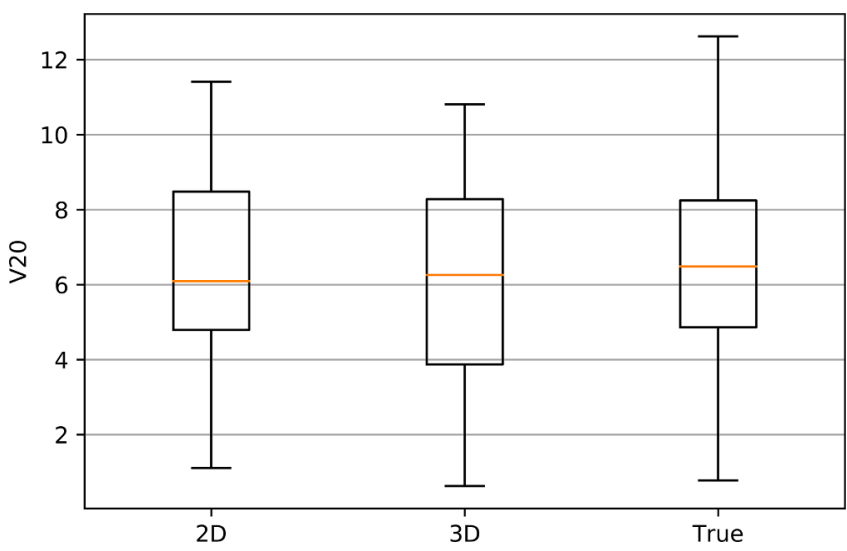

Fig. 7. The V20 was predicted for all 25 patients in the test data set for the $2 \mathrm{D}$ model, 3D model, and displayed with the true V20 distribution from clinically used plans.

The V20 for the 25 test patients is represented as a boxplot in Fig. 7 where we compare the distribution from the $2 \mathrm{D}$ predictions, 3D predictions, and the clinically used plans. The models tend to have lower predicted V20 than the clinically used plans, where the 3D model has a mean of 6.00 and the 2D model has a mean of $6.51(p<0.05)$.

The dose difference for mean dose to each region of interest weighted by the dose prescription for the 25 test patients is represented as a boxplot in Fig. 8. It can be seen that both models tend to predict the mean dose for all regions within $0.05 \%$ of the prescription dose excluding outliers. The average dose differences in mean dose with standard deviation for the 2D model and the 3D model can be found in the appendix as Table A1 where paired sample t-tests were performed. 


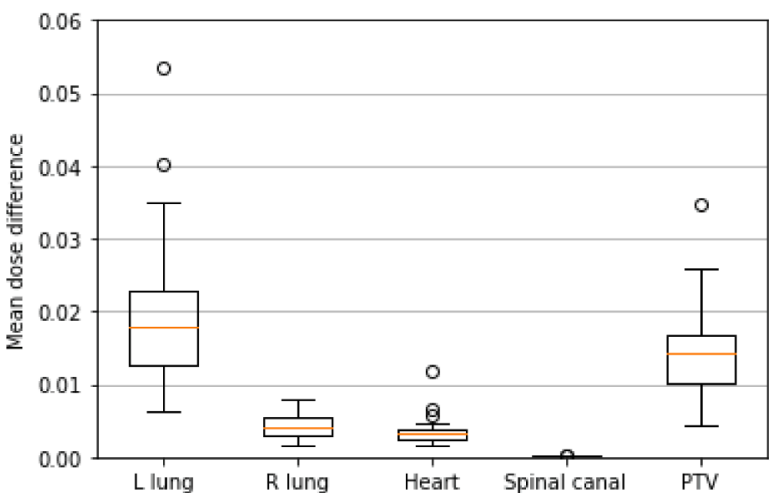

(a)

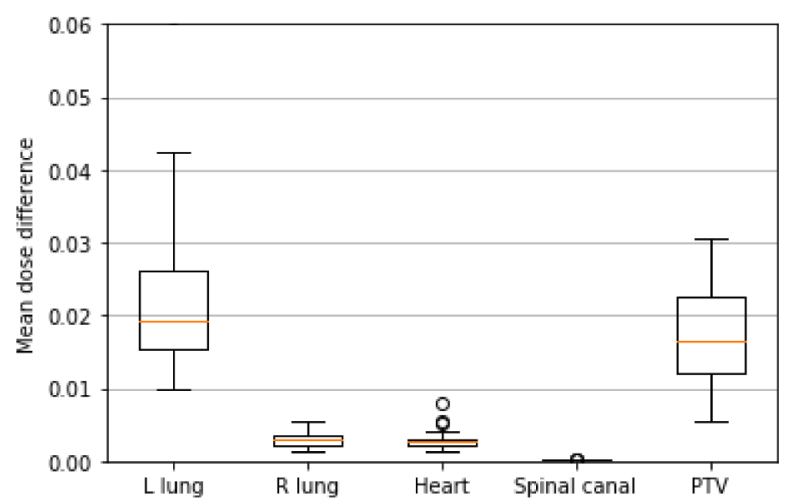

(b)

Fig. 8. The dose difference (\%) for mean dose to each region of interest was predicted for all 25 patients in the test data set for both the (a) 2D model and the (b) 3D model. In this figure, the area covered by the 95\% isodose curve is labeled as 95\% IDL.

The 2D and 3D model perform similarly in predictions on the left lung and spinal canal, with the 3D model performing slightly better on the right lung and heart by $0.001 \%(p<0.05)$.

\section{Discussion}

We have developed two models which successfully can predict the dose distribution for various left-sided breast cancer patients. Overall, the 3D model exceeded the performance of the 2D model which suggests a significance in the spatial information between slices for improving model quality. The loss functions for both models reach a minimum mean squared error around 2 which occurs after about 40 epochs for the 2D model where it then plateaus, while the 3D model reaches the minimum around 180 epochs. This could be due to increased data augmentation in the 3D data as each sub-volume is randomly generated decreasing the number of times the model sees the same portion of the patient volume, and thus requires longer training whereas for the $2 \mathrm{D}$ data, the model always receives a full slice with approximately $30 \%$ randomly augmented so the data is limited to the number of slices.

The distribution of predicted V20 values for the models are very close to that of the V20 from clinically used plans. The model predictions tend to be slightly below the true values, which while they are less accurate compared to those used, could allow for more optimal plans produced with lower doses to the left lung. In comparing the mean V20 over the 25 patients, the 2D model produces predictions closer to those used whereas the 3D model produces predictions with more optimal doses (lower V20).

The average dose difference of mean dose for the 2D model weighted by the dose prescription are also very close to that of the 3D model (Table A1). In terms of mean dose which allows consideration of all pixels or voxels contained within a structure, both models perform very well with the 3D model only slightly better. The results here are also very stable which shows great promise for future clinical implementation of one of the models. In Nguyen et al. (2019), a U-net model is used to predict dose distributions for prostate cancer and the lowest mean dose difference weighted by dose prescription is $0.48 \%$ [5]. Similarly, in Liu et al. 31, a U-ResNet-D model was used to predict dose distributions for nasopharyngeal cancer patients and the lowest mean dose difference seen is $2 \%$ [31]. All values of mean dose difference weighted by dose prescription for our models were less than $0.06 \%$ exhibiting the highlevel of accuracy possible with the given parameters and model architecture.
The 2D model had more trainable parameters with a deeper architecture network, and a larger batch size than the 3D model which should greatly improve the performance of the $2 \mathrm{D}$ model, yet we see that the $3 \mathrm{D}$ model has better results. This suggests that, to some extent, the type of data carries more importance than the depth of the network. Although the 3D model had greater variation, we believe larger batch sizes with longer training could help stabilize the results, however, this would be much more computationally expensive.

With these models, we can quickly produce optimal dose distributions with limited data to be used as a reference for physicians during planning. Due to consistency in performance of the model, using these references would create a new standard of plan quality ensuring consistency in the plans. We can also work towards automated treatment planning to decrease planning times while ensuring patients are always receiving high-quality care. These dose distributions can be used directly with inverse planning to produce clinically acceptable plans. We predict that the models could also, if provided adequate data, be expanded to predict dose distributions for various other cancer sites with similar beam geometry without a loss in performance expanding both the capability and applicability of the models.

\section{Conclusion}

We have demonstrated the effectiveness and importance of implementing machine learning in treatment planning by developing two deep-learning models to predict dose distributions. In exploring the performance of a two-dimensional and three-dimensional model, we have shown that the spatial information given in 3D data can greatly enhance accuracy with the 3D model outperforming the 2D model. Implementation of this model in a clinical setting has great potential to improve plan quality by providing an optimal distribution for reference and in the future, could be used to reduce treatment planning times while maintaining high-quality of plans.

\section{Acknowledgments}

One of the authors N.H. was funded by a Harold E. Johns Studentship provided through Cancer Care Ontario (Ontario Health) and by the Walker Family Cancer Centre. 


\section{Appendix A. Appendix}
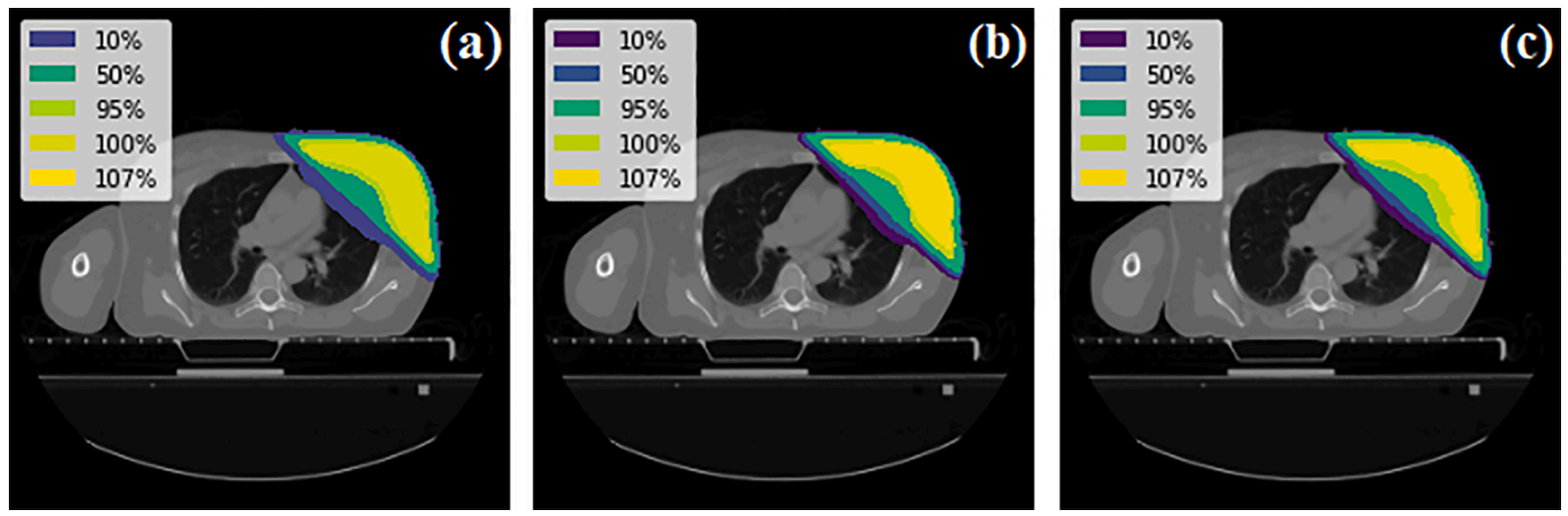

Fig. A1. The (a) 2D, (b) 3D, and (c) clinically used dose distributions weighted by the dose prediction for a random slice of the CT volume of patient B.
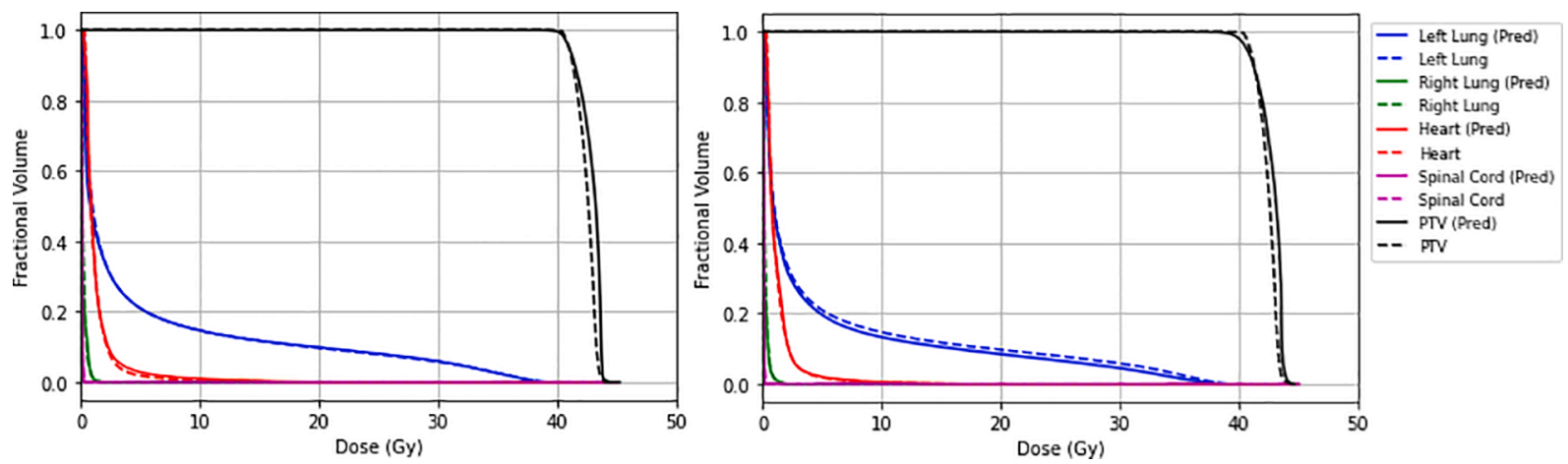

Fig. A2. The cumulative DVH graphs comparing the clinically used results with the 2D model on the left and 3D model on the right for patient $\mathrm{B}$.

\section{Table A1}

Average mean dose difference (\%) and standard deviation over 25 test patients for regions of interest in the two-dimensional and three-dimensional models with the corresponding p-values from paired-samples $t$ test.

\begin{tabular}{lccc}
\hline Structure & $D_{\text {mean }}[2 \mathrm{D}]$ & $D_{\text {mean }}[3 \mathrm{D}]$ & $p$-Value \\
\hline Left lung & $0.02 \pm 0.01$ & $0.02 \pm 0.01$ & 0.009 \\
Right lung & $0.004 \pm 0.002$ & $0.003 \pm 0.001$ & $6.327 \mathrm{E}-08$ \\
Heart & $0.004 \pm 0.002$ & $0.003 \pm 0.001$ & 0.002 \\
Spinal canal & $8.814 \mathrm{E}-05 \pm 3.612 \mathrm{E}-05$ & $7.572 \mathrm{E}-05 \pm 3.530 \mathrm{E}-05$ & 0.01 \\
PTV & $0.015 \pm 0.007$ & $0.017 \pm 0.007$ & 0.0003 \\
\hline
\end{tabular}

\section{References}

[1] Fisher B, Anderson S, Bryant J, Margolese RG, Deutsch M, Fisher ER, et al. Twentyyear follow-up of a randomized trial comparing total mastectomy, lumpectomy, and lumpectomy plus irradiation for the treatment of invasive breast cancer. N Engl J Med 2002;347(16):1233-41.

[2] Clark RM, Whelan T, Levine M, Roberts R, Willan A, McChulloch P, et al. Randomized clinical trial of breast irradiation following lumpectomy and axillary dissection for node-negative breast cancer: an update. J Natl Cancer Inst 1996;88 (22):1659-64.

[3] Veronesi U, Marubini E, Mariani L, Galimberti V, Luini A, Veronesi P, et al. Radiotherapy after breast-preserving surgery in women with localized cancer of the breast. N Engl J Med 1993;328(22):1587-91.

[4] Wang C, Zhu X, Hong JC, Zheng D. Artificial intelligence in radiotherapy treatment planning: present and future. Technol Cancer Res Treat 2019;18. 1533033819873922.
[5] Nguyen D, Long T, Jia X, Lu W, Gu X, Iqbal Z, et al. A feasibility study for predicting optimal radiation therapy dose distributions of prostate cancer patients from patient anatomy using deep learning. Sci Rep 2019;9(1):1-10.

[6] Batumalai V, Jameson MG, Forstner DF, Vial P, Holloway LC. How important is dosimetrist experience for intensity modulated radiation therapy? A comparative analysis of a head and neck case. Pract Radiat Oncol 2013;3(3):e99-106.

[7] Nelms BE, Robinson G, Markham J, Velasco K, Boyd S, Narayan S, et al. Variation in external beam treatment plan quality: an inter-institutional study of planners and planning systems. Pract Radiat Oncol 2012;2(4):296-305.

[8] Jensen AR, Nellemann HM, Overgaard J. Tumor progression in waiting time for radiotherapy in head and neck cancer. Radiother Oncol 2007;84(1):5-10.

[9] Marcello M, Ebert M, Haworth A, Steigler A, Kennedy A, Joseph D, et al. Association between treatment planning and delivery factors and disease progression in prostate cancer radiotherapy: results from the trog 03.04 radar trial. Radiother Oncol 2018;126(2):249-56.

[10] Hebert-Croteau N, Freeman CR, Latreille J, Rivard M, Brisson J. A populationbased study of the impact of delaying radiotherapy after conservative surgery for breast cancer. Breast Cancer Res Treat 2004;88(2):187-96.

[11] Gupta S, King W, Korzeniowski M, Wallace D, Mackillop W. The effect of waiting times for postoperative radiotherapy on outcomes for women receiving partial mastectomy for breast cancer: a systematic review and meta-analysis. Clin Oncol 2016;28(12):739-49.

[12] Benk V, Joseph L, Fortin P, Zhang G, Belisle P, Levinton C, et al. Effect of delay in initiating radiotherapy for patients with early stage breast cancer. Clin Oncol 2004; 16(1):6-11.

[13] Beck RE, Kim L, Yue NJ, Haffty BG, Khan AJ, Goyal S. Treatment techniques to reduce cardiac irradiation for breast cancer patients treated with breast-conserving surgery and radiation therapy: a review. Front Oncol 2014;4:327.

[14] Sardaro A, Petruzzelli MF, D'Errico MP, Grimaldi L, Pili G, Portaluri M. Radiationinduced cardiac damage in early left breast cancer patients: risk factors, biological mechanisms, radiobiology, and dosimetric constraints. Radiother Oncol 2012;103 (2):133-42.

[15] Goody R, O'Hare J, McKenna K, Dearey L, Robinson J, Bell P, et al. Unintended cardiac irradiation during left-sided breast cancer radiotherapy. Br J Radiol 2013; 86(1022):20120434. 
[16] Fan J, Wang J, Chen Z, Hu C, Zhang Z, Hu W. Automatic treatment planning based on three-dimensional dose distribution predicted from deep learning technique. Med Phys 2019;46(1):370-81.

[17] Barragán-Montero AM, Nguyen D, Lu W, Lin MH, Norouzi-Kandalan R, Geets X, et al. Three-dimensional dose prediction for lung IMRT patients with deep neural networks: robust learning from heterogeneous beam configurations. Med Phys 2019;46(8):3679-91.

[18] Nguyen D, Jia X, Sher D, Lin MH, Iqbal Z, Liu H, et al. 3d radiotherapy dose prediction on head and neck cancer patients with a hierarchically densely connected u-net deep learning architecture. Phys Med Biol 2019;64(6):065020.

[19] Sumida I, Magome T, Das IJ, Yamaguchi H, Kizaki H, Aboshi K, et al. A convolution neural network for higher resolution dose prediction in prostate volumetric modulated arc therapy. Phys Med 2020;72:88-95.

[20] Babier A, Mahmood R, McNiven AL, Diamant A, Chan TC. Knowledge-based automated planning with three-dimensional generative adversarial networks. Med Phys 2020;47(2):297-306.

[21] Miki K, Kusters M, Nakashima T, Saito A, Kawahara D, Nishibuchi I, et al. Evaluation of optimization workflow using custom-made planning through predicted dose distribution for head and neck tumor treatment. Phys Med 2020;80: $167-74$.

[22] Fedorov A, Beichel R, Kalpathy-Cramer J, Finet J, Fillion-Robin JC, Pujol S, et al. $3 \mathrm{~d}$ slicer as an image computing platform for the quantitative imaging network. Magn Reson Imaging 2012;30(9):1323-41.
[23] Pinter C, Lasso A, Wang A, Jaffray D, Fichtinger G. Slicerrt - radiation therapy research toolkit for 3D slicer. Med Phys 2012;39(10):6332-8.

[24] Oliphant TE. A guide to NumPy, 1. USA: Trelgol Publishing; 2006.

[25] Van Der Walt S, Colbert SC, Varoquaux G. The numpy array: a structure for efficient numerical computation. Comput Sci Eng 2011;13(2):22.

[26] Mason, D, et al. pydicom: An open source dicom library (Jan. 2019). https://doi. org/10.5281/zenodo.2541240. URL: https://github.com/pydicom/pydicom.

[27] Ronneberger O, Fischer P, Brox T. U-net: Convolutional networks for biomedical image segmentation, in: Medical Image Computing and Computer-Assisted Intervention (MICCAI), Vol. 9351 of LNCS, Springer, 2015, pp. 234-241. URL: htt p://lmb.informatik.uni-freiburg.de/Publications/2015/RFB15a.

[28] Kingma DP, Ba J. Adam: A method for stochastic optimization, arXiv (2014) 1412.6980 .

[29] Chollet F. et al. Keras, URL: https://keras.io (2015).

[30] Abadi M, Barham P, Chen J, Chen Z, Davis A, Dean J, et al. Tensorflow: A system for large-scale machine learning. In: 12th USENIX Symposium on Operating Systems Design and Implementation (OSDI 16); 2016. p. 265-83. URL: https://www.usenix.org/system/files/conference/osdi16/osdi16-abadi.pdf.

[31] Liu Z, Fan J, Li M, Yan H, Hu Z, Huang P, et al. A deep learning method for prediction of three-dimensional dose distribution of helical tomotherapy. Med Phys 2019;46(5):1972-83. 